\title{
Tumor Lysis Syndrome from a Solitary Nonseminomatous Germ Cell Tumor
}

\author{
Brandon T. Nokes, $M D^{1}$ \\ Rodrigo Cartin-Ceba, $\mathrm{MD}^{2}$ \\ Joseph Farmer, MD2 \\ Alyssa B. Chapital, MD, PhD' \\ ${ }^{1}$ Hospital Internal Medicine and ${ }^{2}$ Division of Critical Care \\ Mayo Clinic Arizona \\ Phoenix, AZ USA
}

\begin{abstract}
Spontaneous tumor lysis syndrome is a rare clinical entity, which typically occurs in the context of rapidly proliferating hematologic malignancies. Tumor lysis syndrome in solid organ malignancies is even rarer, and typically provoked by cytotoxic treatment regimens. We describe a case of spontaneous tumor lysis of a solitary metastatic brain lesion from a nonseminomatous germ cell tumor. This case is unique in that spontaneous tumor lysis from a brain metastasis of a solid organ malignancy has never been reported, and spontaneous tumor lysis in a nonseminomatous germ cell tumor is exceedingly rare.
\end{abstract}

\section{Case Report}

A 31-year-old gentleman was admitted to our facility after developing status epilepticus and consequently, being involved in a MVA. Imaging revealed a $3.5 \mathrm{~cm}$ right frontal brain lesion with surrounding edema, but no other acute intracranial pathology. The patient was intubated, sedated, and transferred to critical care for further treatment. His past medical history was notable for primary surgical resection of a T1N0M0 nonseminomatous germ cell tumor in March 2015, followed by detection of a $2.5 \mathrm{~cm}$ lung nodule in September 2015, with concurrent beta-human chorionic gonadotropin (HCG) and alpha-fetoprotein (AFP) biochemical recurrence. He underwent 4 cycles of bleomycin, etoposide, and cisplatin (BEP).

A head CT revealed a $4 \mathrm{~cm} \times 3.5 \mathrm{~cm}$ right frontal lesion with surrounding edema (Figure 1). 


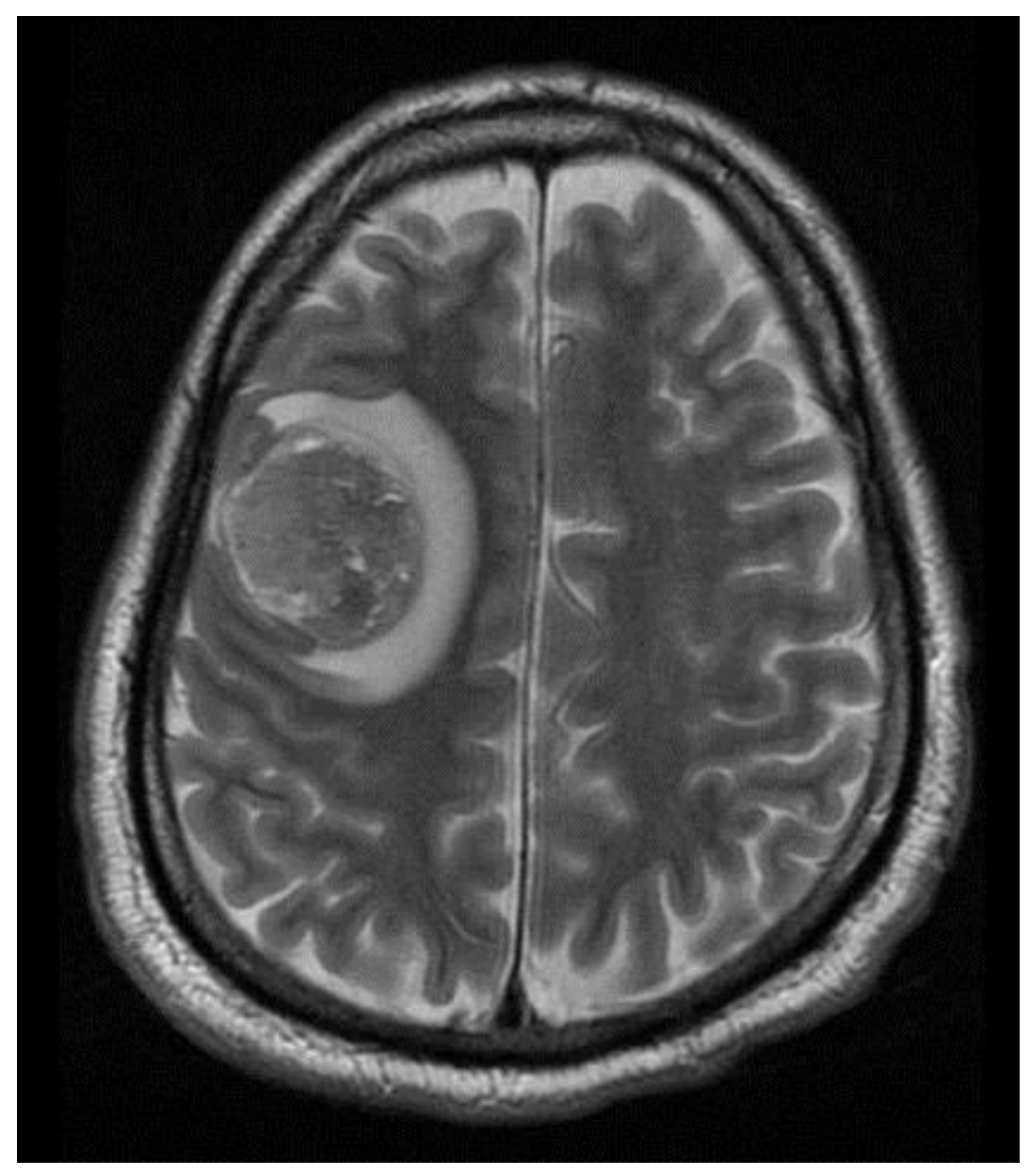

Figure 1. T2 Axial MRI showing $4 \mathrm{~cm} \times 3.5 \mathrm{~cm}$ lesion with associated vasogenic edema.

Dexamethasone $4 \mathrm{mg}$ every 6 hours was initiated for treatment of vasogenic edema. Laboratory studies were significant for a white blood cell count elevated at $19.3 \times 10 \% / \mathrm{L}$, international normalized ratio (INR) 1.34, partial thromboplastin time (PTT) 26.2 seconds, and prothrombin time (PT) 16.1 seconds. Plasma lactate was elevated at $30.6 \mathrm{mmol} / \mathrm{L}$. Bicarbonate was $6 \mathrm{mmol} / \mathrm{L}$ with an anion gap of 45 , glucose $186 \mathrm{mg} / \mathrm{dL}$, BUN $15.2 \mathrm{mg} / \mathrm{dL}$, and creatinine was $2.0 \mathrm{mg} / \mathrm{dL}$. Urine drug screen was negative. His AFP was $7.4 \mathrm{ng} / \mathrm{mL}$ and beta-HCG was $13 \mathrm{IU} / \mathrm{L}$. Over the following 24 hours, the patient experienced decreased urine output. A bedside ultrasound reveals normal IVC collapse. Further lab assessment revealed a CK within normal limits and a urinalysis showed the presence of 11 to $20 \mathrm{RBCs}, 4$ to $10 \mathrm{WBC}$ and some granular casts as well as trace protein. His phosphorus was 8.9 , calcium 8.1 , and uric acid was $13 \mathrm{mg} / \mathrm{dL}$. His lactate dehydrogenase levels were also elevated at $271 \mathrm{U} / \mathrm{L}$.

Due to concern of tumor lysis syndrome, the patient was initiated on rasburicase, which was followed by maintenance allopurinol $300 \mathrm{mg}$ daily. However, due to worsening renal failure, the patient was started on hemodialysis. He was taken to the operating room the following morning for immediate surgical resection of his brain metastasis; no evidence of residual disease was seen on follow-up imaging (Figure 2). 


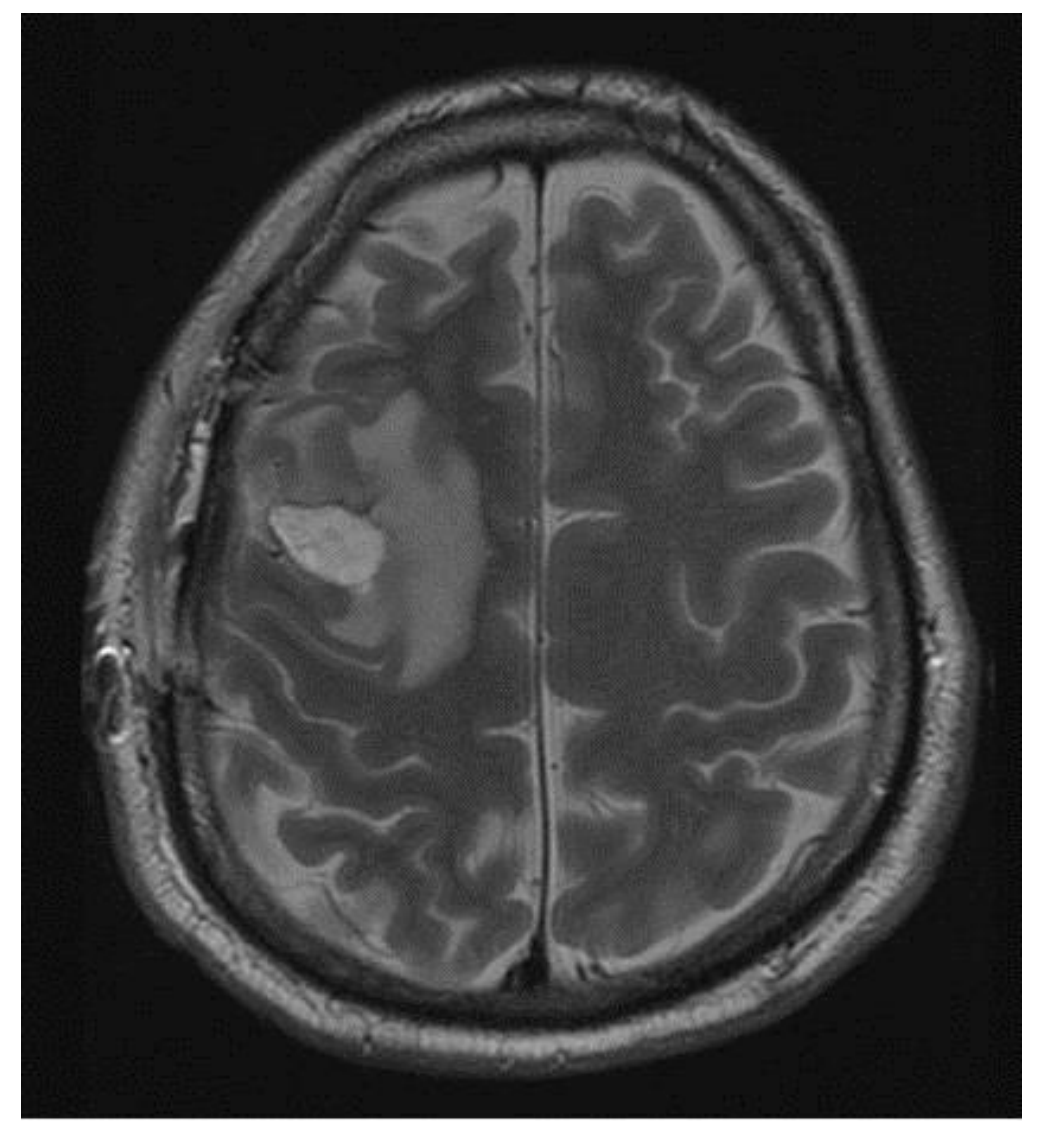

Figure 2. T2 Axial MRI status post a right frontal craniotomy and gross total resection of the previously noted mass. Small amount of blood noted within the resection cavity. Residual vasogenic edema persists in the white matter surrounding the operative bed.

Repeat chest, abdomen and pelvis imaging did not show any additional metastatic lesions.

In the following days, he was subsequently extubated, transferred to the floor, and continued hemodialysis, eventually fully recovering his renal function. Ultimately, he was discharged with outpatient follow-up for additional chemotherapy planning after physical rehabilitation.

\section{Discussion}

Tumor lysis syndrome (TLS) can be subdivided into laboratory TLS and clinical TLS, as defined by the Cairo-Bishop diagnostic criteria (1). Spontaneous TLS can occur in solid organ malignancies (1). TLS in solid organ malignancies is provoked by chemotherapy or radiation therapy, which creates massive cell lysis and elaboration of intracellular potassium, phosphate, and uric acid as well as hypocalcemia, which can lead to renal failure and cardiac dysrhythmias (1). LDH is also elevated. TLS can also be thought of as being provoked, either by ongoing chemotherapy or a decrease in effective circulating volume, or unprovoked. It is rare for TLS to occur in nonseminomatous germ 
cell tumors. Only 2 case reports have been published regarding spontaneous TLS in nonseminomatous germ cell tumors $(2,3)$. Our case is most likely a spontaneous TLS. To date, no reports have been published regarding spontaneous TLS from a solitary brain metastasis from a nonseminomatous germ cell tumor. Further, no cases have been reported regarding tumor lysis from a solitary brain metastasis of any solid organ malignancy.

The occurrence of TLS in solid organ malignancies is thought to occur secondary to rapid cellular proliferation that exceeds the available blood supply for a tumor, leading to tumor ischemia and diffuse tumor cell necrosis. The biochemical milieu elaborated from these necrotic cells can result in end-organ pathology.

The treatment of TLS is contingent upon the rate of cancer progression and whether there is evidence of end-organ damage. Importantly and ideally, patients can be stratified into intermediate, moderate, or high-risk of developing TLS based on their malignancy type and rate of cancer progression, such that TLS may be prevented with prophylactic hydration, electrolyte monitoring and allopurinol or rasburicase $(4,5)$. Biochemical TLS alone can be treated with IV hydration and allopurinol, a xanthine oxidase inhibitor which potentially halts TLS progression. When there is end-organ damage, rasburicase (a recombinant urate oxidase) is the first-line treatment along with aggressive hydration (5). Additional therapies are directed towards minimizing sequelae of TLS (i.e. calcium gluconate for hyperkalemia associated EKG changes or emergent dialysis for acute renal failure). There is no role for urinary alkalinization.

We were fortunate in that our patient had a great outcome, owing to early detection and aggressive intervention, and we implore our fellow physicians to be mindful of TLS as a possible clinical outcome in all malignancies, irrespective of its clinical rarity.

\section{References}

1. Mirrakhimov AE, Ali AM, Khan M, Barbaryan A. Tumor lysis syndrome in solid tumors: an up to date review of the literature. Rare Tumors. 2014;6(2):5389. [CrossRef] [PubMed]

2. D'Alessandro V, Greco A, Clemente C, et al. Severe spontaneous acute tumor lysis syndrome and hypoglycemia in patient with germ cell tumor. Tumori. 2010;96(6):1040-3. [PubMed]

3. Pentheroudakis G, O'Neill VJ, Vasey P, Kaye SB. Spontaneous acute tumour lysis syndrome in patients with metastatic germ cell tumours. Report of two cases. Support Care Cancer. 2001;9(7):554-7. [CrossRef] [PubMed]

4. Feres GA, Salluh JI, Ferreira CG, Soares M. Severe acute tumor lysis syndrome in patients with germ-cell tumors. Indian J Urol. 2008;24(4):555-7. [CrossRef] [PubMed]

5. Coiffier B, Altman A, Pui CH, Younes A, Cairo MS. Guidelines for the management of pediatric and adult tumor lysis syndrome: an evidence-based review. J Clin Oncol. 2008;26(16): 2767-78. [CrossRef] [PubMed] 